\title{
Modelling the Translational Dynamics of the Atlas Motion Platform
}

\author{
Kaitlin Leigh ${ }^{1}$, Michael Rappoport ${ }^{1}$, Jerome Thevenot ${ }^{1}$, Robert Langlois ${ }^{1}$ \\ ${ }^{1}$ Department of Mechanical and Aerospace Engineering, Carleton University \\ 1125 Colonel By Drive, Ottawa, Canada \\ KaitlinLeigh@cmail.carleton.ca; MichaelRappoport@cmail.carleton.ca; JeromeThevenot@cmail.carleton.ca; \\ Robert.Langlois@carleton.ca
}

\begin{abstract}
The novel Atlas motion platform is designed to prescribe in six degrees of freedom the motion of a spherical simulator cockpit with the unusual capability of providing kinematically-uncoupled translational and rotational motion, unbounded rotational motion, and a fully-dexterous workspace. In support of the design and operation of the Atlas simulator, a translational dynamic model of the system has been developed. It includes the prescribed motion of the interface between the translational and rotational stages, translational motion of the spherical cockpit, and translational motion of the upper 'halo' support structure. Contact points between the spherical cockpit and the constraint/actuation system were modelled using directed elements consisting of either constant-force elements or spring/damper elements. In the case of the spring/damper elements, stiffness and damping parameters were obtained by a combination of finite element analysis of structural elements and experimental characterization of viscoelastic elements along the interfacing load paths. The dynamic model is suitable for evaluating internal forces and relative motions within the simulator resulting from translation motions; and forms the basis for extension to include rotational dynamics of the simulator system.
\end{abstract}

Keywords: Atlas motion platform, dynamic modelling, effective stiffness, finite element analysis, material characterization

\section{Introduction}

The Carleton University Simulator Project (CUSP) is a final-year engineering capstone project that began in 2002. CUSP is focused on developing an innovative flight simulator capable of unbounded rotation that will be able to more closely replicate edge-of-envelope flight conditions than conventional flight simulators. The resulting novel full-scale flight simulator is called Atlas and is illustrated in Figure 1. It's actuation system produces three translational degrees of freedom and three rotational degrees of freedom that are kinematically uncoupled from translation and allow unbounded rotation [1]. Further, because translation and rotation are uncoupled, the workspace of the device is fully dexterous. The cockpit of the simulator is contained within a $9.5 \mathrm{ft}$ diameter sphere which is driven by three active mecanum wheels [2]. There are six sets of two passive mecanum wheels that support the weight of the sphere and constrain its motion relative to the interface platform at the base of the rotational stage. Above the sphere rests the halo which interfaces with the sphere through twelve passive mecanum wheels in order to constrain the upper portion of the sphere during motion as well as to passively provide downward force on the sphere to react the contact forces produced by the active mecanum wheels which drive the rotation. The entire rotational stage assembly is attached to the flying frame of a MOOG Stewart-Gough motion base that is used to provide the translational degrees of freedom for the system. At the present time, the system is in its final stages of assembly with commissioning expected to be completed in 2018.

Ensuring adequate performance and safety of the Atlas simulator relies on the availability of an accurate dynamic model. The model can be used for determining peak and fatigue loading on various structural components, supporting rigidbody vibration analysis of the system, determining operating parameters to ensure sufficient tractive force at the driving wheel contact points, and supporting development of the simulator control laws. Further, given that the simulator will be used for a variety of research purposes for which distinctly-different cockpit configurations (and hence different inertial properties) may be required and a variety of prescribed and interactive motion algorithms may be used, it is prudent to have the ability to simulate runs in a virtual environment before running them on the physical system with a passenger/trainee in the cockpit. For this purpose, the dynamic model will be interfaced to the Atlas computing architecture to provide a stand-in for the physical system for such virtual evaluations.

This paper is structured such that the dynamic modelling approach is presented first, followed by structural modelling that was required to obtain the effective stiffness of structural components, followed by experimental testing that was undertaken to characterize the properties of the viscoelastic castor rollers that form the contact points with the sphere. Following the development of the model, observations, conclusions, and an indication of future work are presented.

\section{Dynamic Model}

In general, the dynamic model should include six-degree-of-freedom motion of the interface platform (that moves with the flying frame of the motion base); translational and rotational motion of the sphere, and translational and rotational motion 


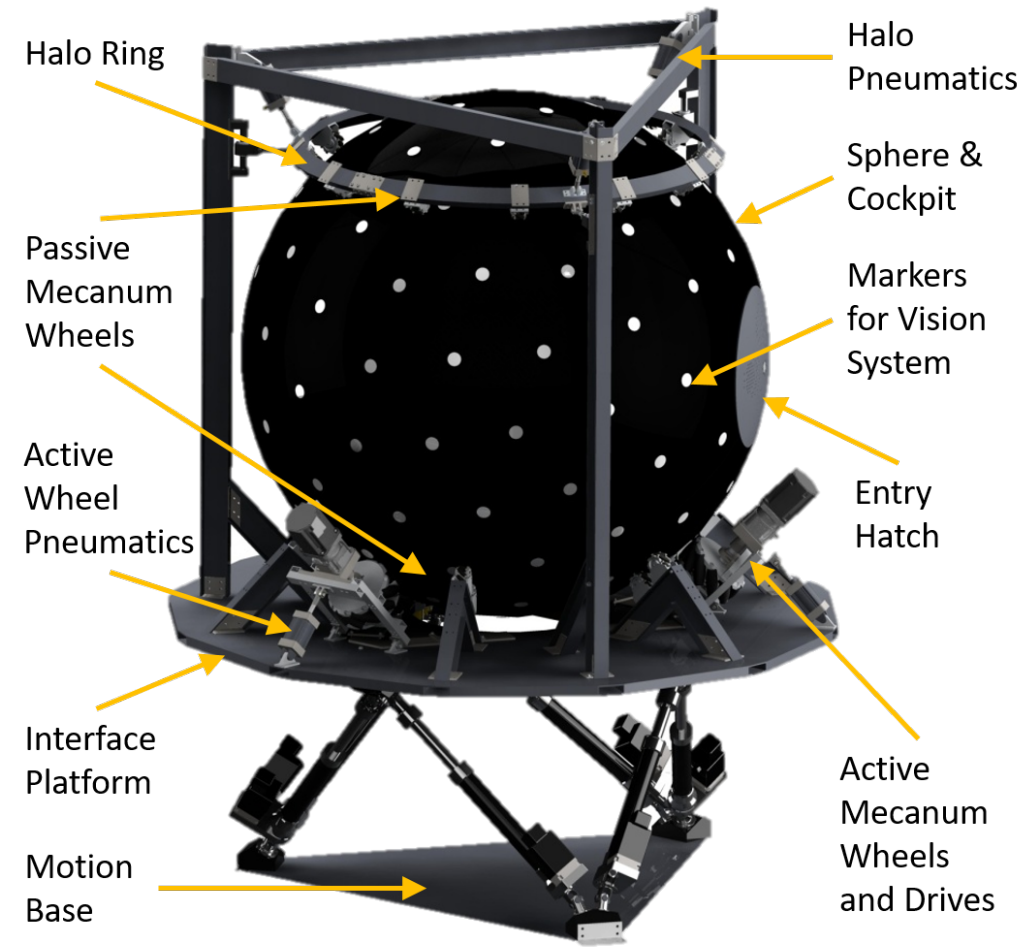

Fig. 1: Computer-aided design representation of the full-scale Atlas simulator.

of the halo assembly. Even though the motion base drive algorithm is theoretically constrained in software to pure translational motion, the possible undesirable angular motions resulting from malfunction of one or more of the motion base prismatic actuators or the drive algorithm should be included. The focus of this paper is on the translational portion of the rigid-body dynamic model. As with all aspects of this primarily-undergraduate design project, developments are made incrementally in clearly-defined steps whenever practical. Further, the structural response of the system is investigated separately using a dedicated finite-element-based structural dynamics model [3].

Referring to Figure 1, it is apparent that, at minimum, a rigid-body translational dynamic model of the system must include the prescribed translational motion of the interface platform and translational motion of the sphere. Such a model would contain six degrees of freedom with the three degrees of freedom representing the interface platform motion being prescribed. Such a model is represented schematically in Figure 2. Recognizing compliance of the upper passive wheels, it is possible for the halo assembly to move relative to the sphere and upper support structure (that is assumed to have the same motion as the interface platform) for the purpose of the rigid-body model. To accommodate this, the six-degree-offreedom dynamic model was extended to include the translational motion of the halo, resulting in three additional degrees of freedom. The resulting model contains a total of nine translational degrees of freedom (including the three prescribed degrees of freedom of the interface platform) and is illustrated schematically in Figure 3.

Figures 2 and 3 show a number of springs representing directed force-producing elements at contact points between the interface platform, halo, and sphere where the magnitude of the interface force is dependent on the relative positions between contact points (spring attachment points) as well as the corresponding relative velocities between those points. A pneumatic system controls the air pressure in the pneumatic actuators that provide downward force on the halo as well as those that press the active mecanum wheels against the sphere surface. The currently-envisioned operational scenario is to have the upper (halo) pneumatics pressurized and then sealed such that the three cylinders act as precompressed air springs; and to have the lower (active mecanum wheel) pneumatic pressures actively controlled such that the contact forces with the sphere remain constant. As a result, in total, the model contains 18 spring and damper directed elements representing all of the 24 passive wheel contact points with the sphere, 3 spring and damper elements representing the halo pneumatic cylinders, and 3 constant-force directed elements that represent the active wheel pivot assemblies that apply forces to the sphere at the active wheel contact points. Each contact point between the halo and sphere is modelled by one element (12 in total) and each pair of contact points on the lower passive wheels is modelled as one element (6).

Each of the spring forces $F_{S_{i}}$ was evaluated using

$$
\vec{F}_{S_{i}}=\hat{r}_{b / a}\left(k_{\text {effective }_{i}}\left(\left\|\vec{r}_{b / a}\right\|-l_{0_{i}}\right)\right)
$$



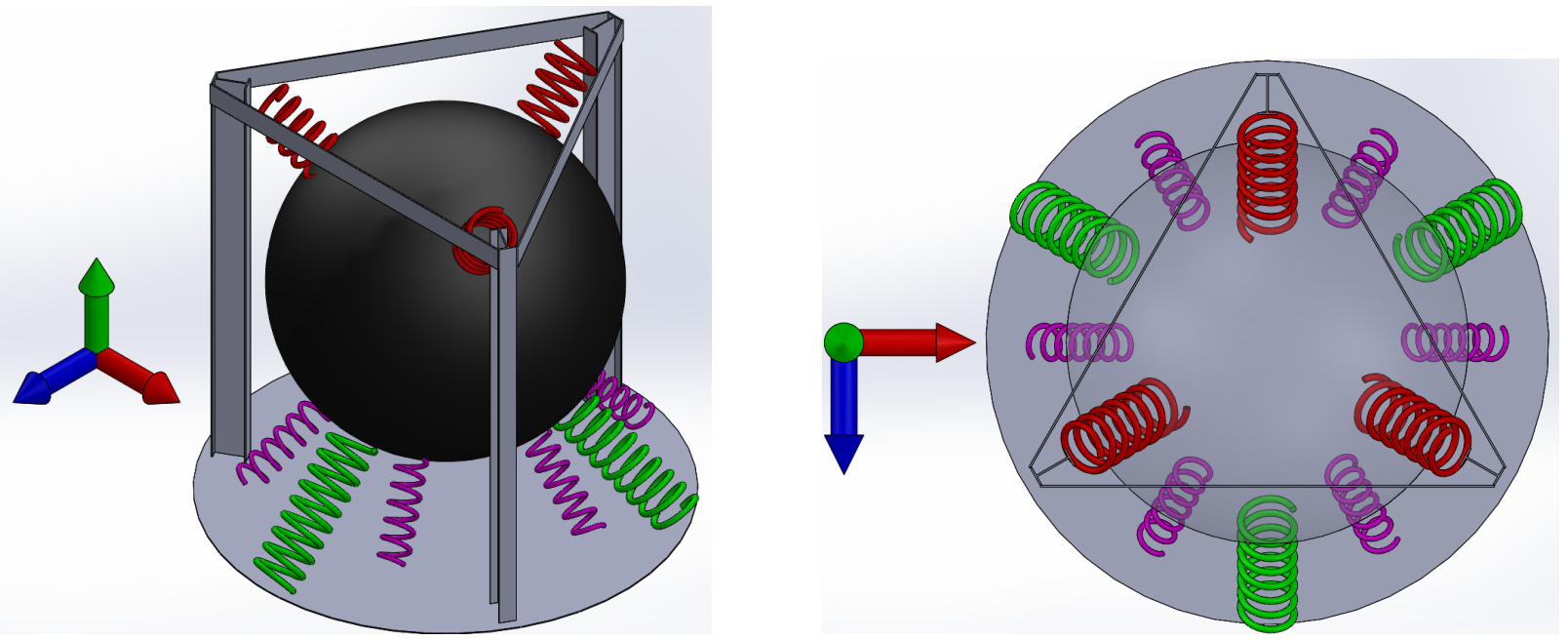

Fig. 2: Six-degree-of-freedom dynamic model as viewed from the front (left) and top (right).
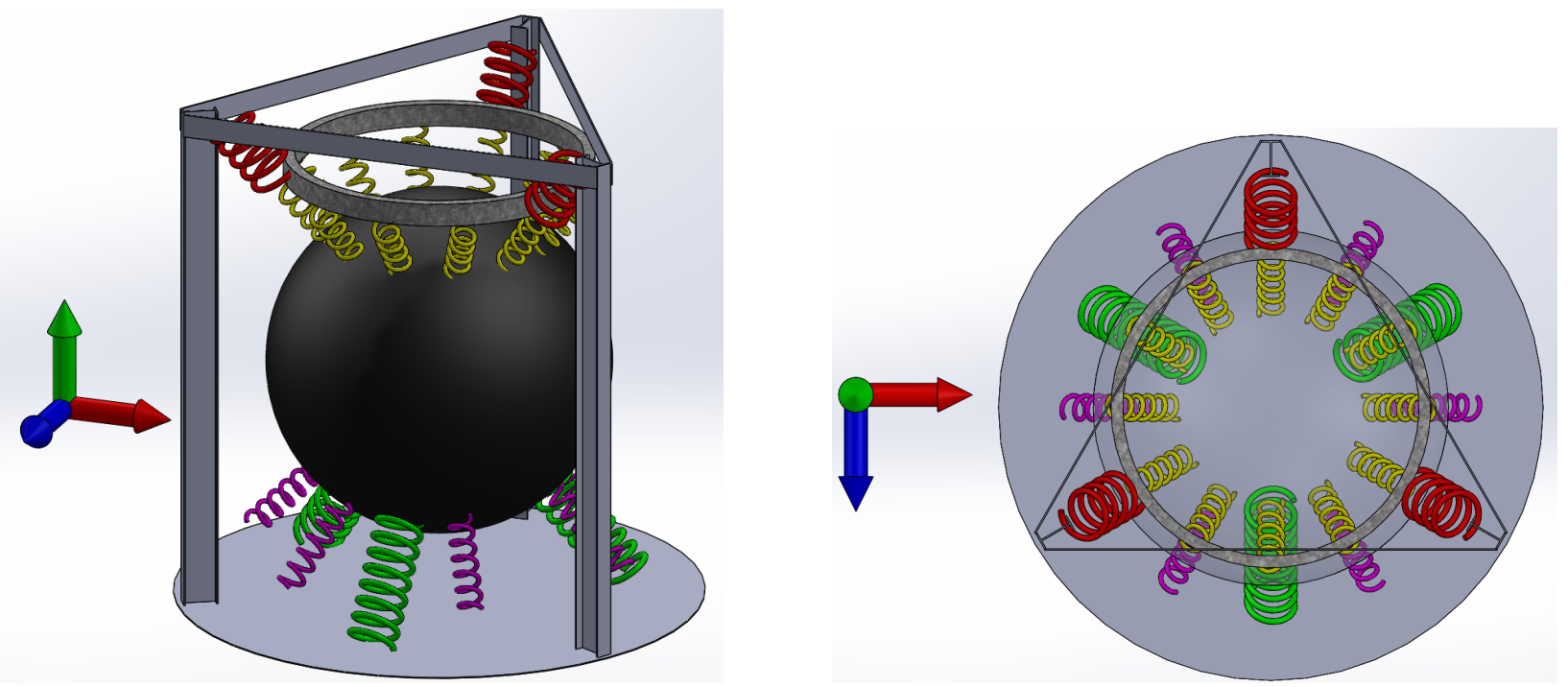

Fig. 3: Nine-degree-of-freedom dynamic model as viewed from the front (left) and top (right).

where $i$ is the index referring to a specific directed elastic element, $\vec{r}_{b / a}$ is a vector directed from elastic element attachment point $a$ to elastic element attachment point $b, \hat{r}_{b / a}$ is the unit vector corresponding to $\vec{r}_{b / a}, k_{\text {effective }}$ is the effective stiffness of the components represented by the elastic element, and $l_{0_{i}}$ is the unstretched length of the components represented by the elastic element.

The damping component of an elastic element directed force $F_{D_{i}}$ is evaluated using

$$
\vec{F}_{D_{i}}=\hat{r}_{b / a}\left(c_{\text {effective }_{i}}\left(\vec{v}_{b / a} \cdot \hat{r}_{b / a}\right)\right)
$$

where $c_{\text {effective }}$ is an effective viscous damping coefficient and $\vec{v}_{b / a}$ is the velocity of attachment point $b$ relative to attachment point $a$.

The overall elastic directed element forces $F_{i}$ are evaluated by summing the stiffness and damping contributions

$$
\vec{F}_{i}=\vec{F}_{S_{i}}+\vec{F}_{D_{i}}
$$

Based on Newton's third law, these forces are positive as they act at points $a$ and negative as they act at points $b$.

In the case of the constant-force elements, while the magnitude of the force remains constant, the direction of the force is dependent upon the relative positions of the contact points such that

$$
\vec{F}_{C_{j}}=\hat{r}_{b / a} F_{j}
$$


where $j$ is the index corresponding to a particular constant-force element and $F_{j}$ is the magnitude of the constant force. Again, these are interpreted as being positive at point $a$ and negative at point $b$.

The translational kinematics of the system are expressed using 9 generalized coordinates: 3 absolute orthogonal displacements of the interface platform, 3 absolute orthogonal displacements of the sphere, and 3 orthogonal displacements of the halo ring. All of the relative position and relative velocity vectors needed to evaluate the forces acting on the bodies are evaluated from the system state vector comprised of the 9 generalized coordinates and their first time derivatives.

Knowing all of the force components acting on the halo and sphere assemblies, Newton's law is applied separately to the sphere and halo bodies resulting in the accelerations of each of those two bodies expressed in terms of the second derivatives of the sphere and halo generalized coordinates.

Numerical integration results in updated estimates of the sphere and halo absolute positions and velocities, from which the system forces can again be evaluated. This process repeats, thereby propagating the translational motions of the simulator forward in time and evaluating all of the relevant internal forces.

The effective stiffness and damping characteristics captured by each of the elastic directed elements are based on consideration of the particular components that act between interface points $a$ and $b$. The effective stiffness model for the lower passive support structures is illustrated in Figure 4. The effective stiffness comprises the structural stiffness of the aluminum base bracket, the stiffness of the roller support structure, and the stiffness of the two passive mecanum wheel castor/axle assemblies that contact the sphere; with all of these elements arranged as springs in either series or parallel corresponding to the real system as indicated in Figure 4. Similarly, the stiffness model of the upper passive wheels is illustrated in Figure 6.
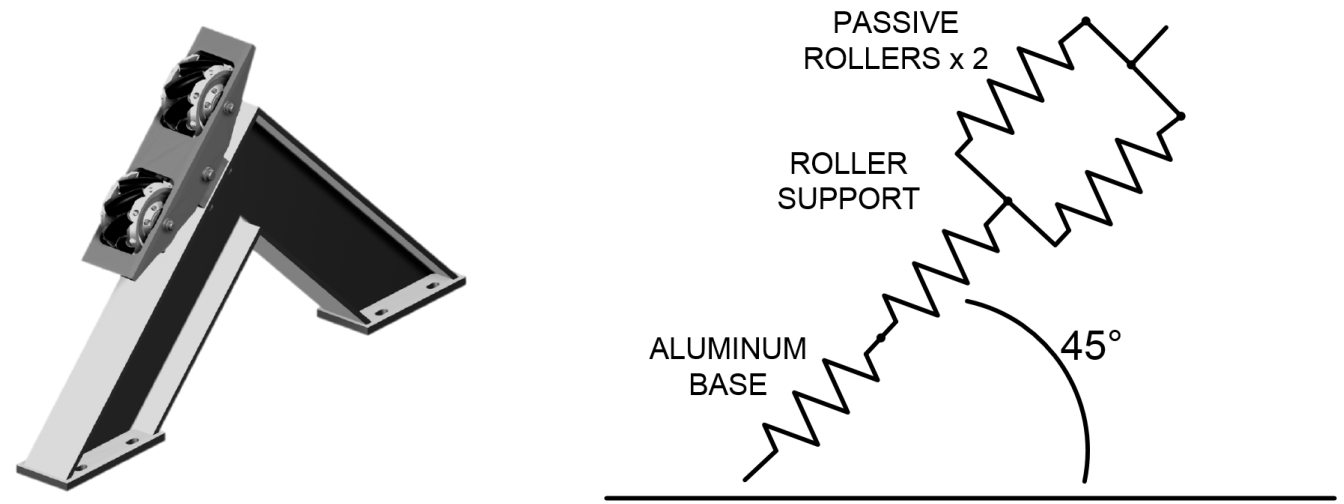

Fig. 4: Lower passive wheel support structure (left) and schematic representation (right).

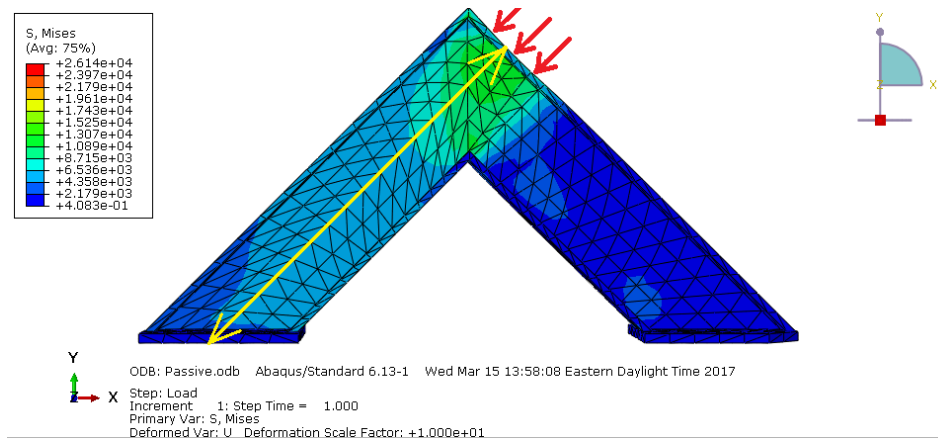

Fig. 5: FEA results of lower passive wheels.

For both the lower and upper passive wheel structures, the structural damping of the metal structures was considered to be negligible relative to the damping associated with the viscoelastic deformation of the castor wheel urethane. As such, the effective damping values came directly for the material testing presented subsequently.

\section{Structural Components}

The flexibility of structural components along the load paths that impact the dynamic model need to be incorporated into the effective stiffnesses characterizing the dynamic model. The approach involved assuming that the structural components connecting rigid bodies act as equivalent springs through which forces are transmitted. The intermediate components were 

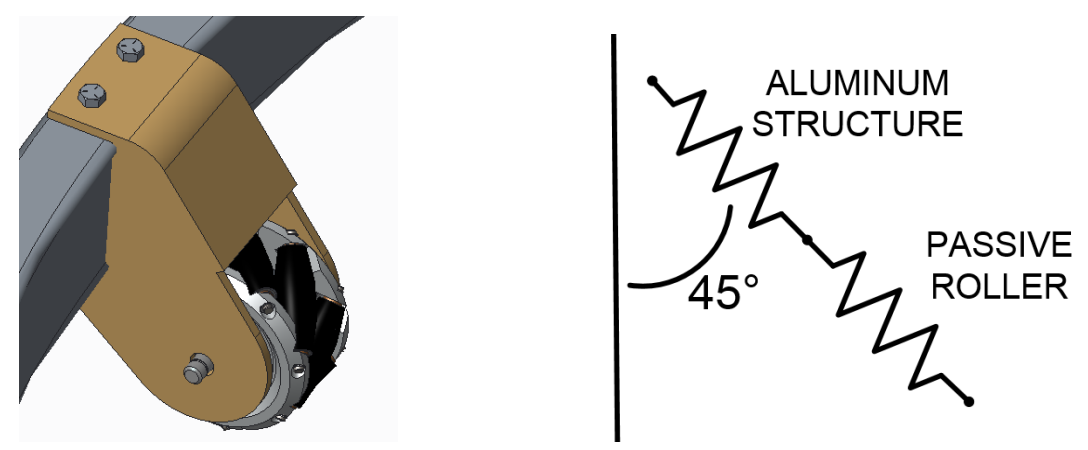

Fig. 6: Upper passive wheel support structure (left) and schematic representation (right).

divided into subcomponents in order to analyze the model as a spring-damper system. A spring constant was calculated for each subcomponent. Using traditional series and parallel spring calculations, the equivalent spring constant was determined for the complete component assembly [4].

Through the use of finite element analysis (FEA), the deflections of components corresponding to known loads were determined. The collected data was subsequently used to calculate equivalent spring constants $k_{i}$ where

$$
k_{i}=F_{\text {applied }} / \Delta x_{i}
$$

where $k_{i}$ is the $i^{\text {th }}$ spring stiffness, $F_{\text {applied }}$ is the applied force, and $\Delta x_{i}$ is the $i^{\text {th }}$ displacement. In applying this, both the force and displacement are taken to be in the direction along which the stiffness is required.

\section{Lower Passive Wheels}

The lower passive wheels were modelled as a spring system as shown in Figure 4. The spring system includes two passive rollers, the roller support, and the aluminum base.

In order to determine the spring constant for the aluminum base, the passive wheel mount was analyzed using FEA in Abaqus/CAE [5]. The component was meshed using quadratic tetrahedral elements of type C3D10. The base was constrained at the bolt holes in all directions. A load of $1000 \mathrm{lb}_{\mathrm{f}}$ was applied to the passive mount as shown in Figure 5. A deflection of 0.0143 in was measured along the line of action of the load at $45^{\circ}$. This resulted in a spring constant of $1.17 \times 10^{7} \mathrm{~N} / \mathrm{m}$. Note that both SI and Imperial units are used reflecting the need to perform the mechanical design in Imperial units consistent available stock material sizes and manufacturing resources; while analysis is performed using SI for convenience.

The roller support was modelled in Creo Simulate [6] as shown in Figure 7 (left) [7]. A load of $569 \mathrm{lb}_{\mathrm{f}}$ was applied leading to a measured deflection of $1.31 \times 10^{-3}$ in. This resulted in a spring constant of $6.68 \times 10^{7} \mathrm{~N} / \mathrm{m}$.

\section{Halo Passive Wheels}

The halo passive wheels were modelled as a spring system as shown in Figure 6. The spring system includes the passive roller and the aluminum structure.

The spring constant for the aluminum structure was determined using FEA in Abaqus/CAE [5] (Figure 7 (right)). The component was meshed using quadratic tetrahedral elements of type C3D10. The structure was constrained at the bolt holes in all directions. A load of $540 \mathrm{lb}_{\mathrm{f}}$ was applied to the aluminum structure. A deflection of $1 \times 10^{-5}$ in was measured along the line of action of the load at $45^{\circ}$. This resulted in a spring constant of $2.40 \times 10^{8} \mathrm{~N} / \mathrm{m}$.

\section{Mecanum Roller Properties}

Accurate modelling of the Atlas motion platform requires effective stiffness values to represent the current design of both the active and passive mecanum wheel rollers. Both sizes of castor rollers have gone through several iterations to achieve rollers that can withstand the required loads while remaining firmly adhered to the roller shaft and while also providing a contact patch at the interface with the sphere that is adequate in size such that the bearing pressure does not exceed the allowable sphere contact pressure. As such, the material spring constants and damping curves for the rollers required investigation. Several experiments were conducted to characterize the properties of the mecanum wheels for this purpose. A Material Testing System (MTS) and a hydraulic press were utilized to subject an individual roller of each type of mecanum wheel to monotonic and cyclic loading. The MTS was operated using force control for determining the specific material properties that derived from both monotonic and cyclic load testing.

\section{Monotonic Loading}

In the case of monotonic loading, the MTS was calibrated to compress each roller in the direction perpendicular to the roller axis at the theoretical worst-case loading condition that could be experienced during the course of a routine simulation. 

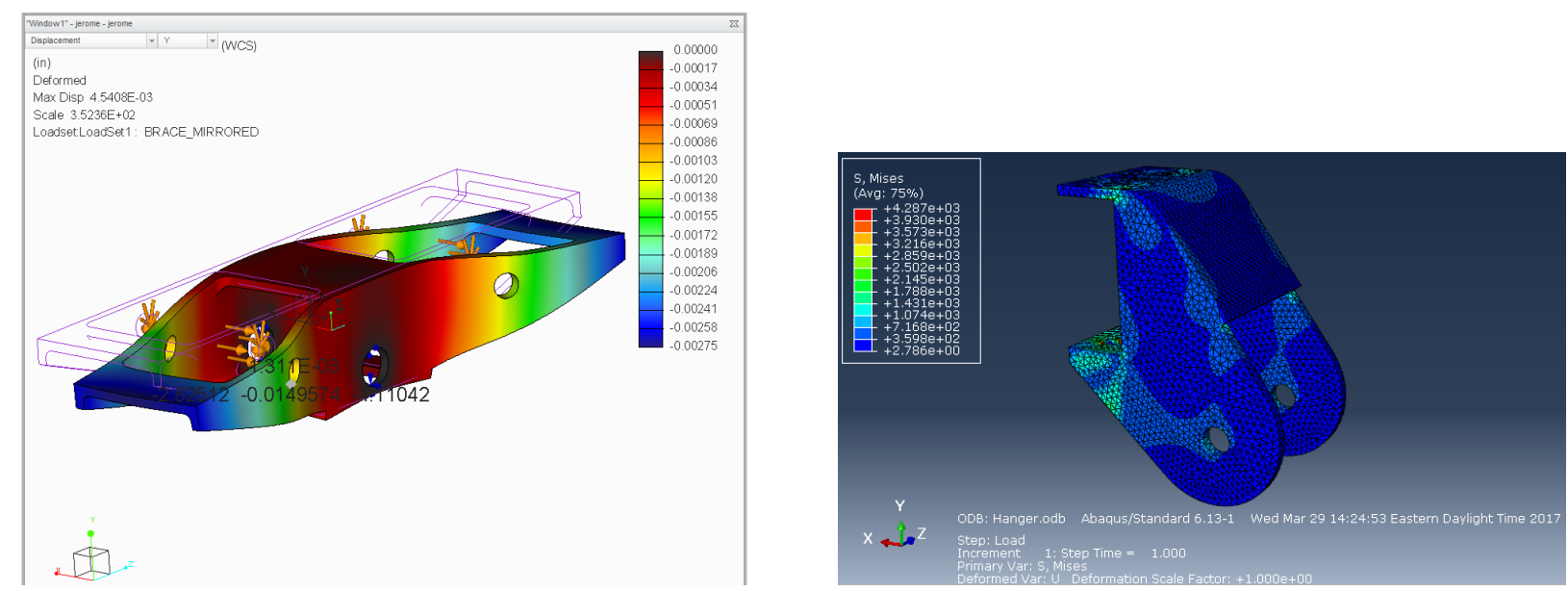

Fig. 7: FEA results of lower roller support (left) and halo support bracket for the passive mecanum wheels.

The worst-case normal loads for the passive and active rollers are $250 \mathrm{lb}_{\mathrm{f}}$ and $1500 \mathrm{lb}_{\mathrm{f}}$ respectively. The number of specimens tested was limited by the availability of excess rollers not in-use on the prototype Atlas simulator. This meant that tests were conducted on two passive rollers and one active roller during the experiment. Circumferential uniformity and consistency of each roller's properties were investigated by rotating each specimen by $90^{\circ}$ after each measurement. For each trial, the applied load and displacement of the MTS frame were recorded. The displacement of the frame corresponds to the displacement of the polyurethane layer on the mecanum wheel rollers. The data were then plotted and an example can be seen in Figure 8 for a passive roller. The spring stiffness was determined from the slope of the force-displacement curve at each position and then averaged. The initial horizontal portion of the curve represents the MTS stroke prior to contacting the surface of the specimen [8].

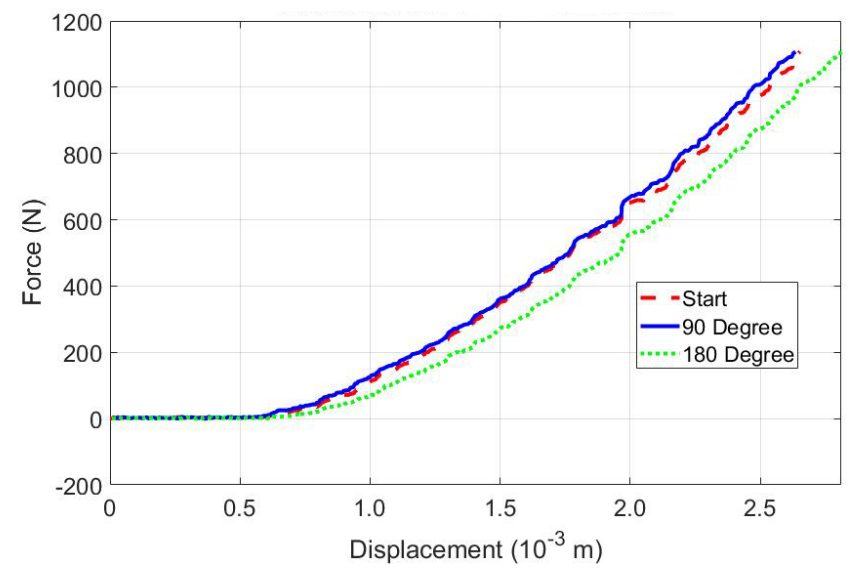

Fig. 8: Passive roller monotonic loading result.

\section{Cyclic Loading}

An initial investigation was performed into the damping characteristics of the mecanum rollers. The same specimens were utilized from the monotonic experiment. Each roller was subjected to cyclic loading at several frequencies: $1 \mathrm{~Hz}, 3 \mathrm{~Hz}$, and $5 \mathrm{~Hz}$. The MTS was calibrated to run for 100 cycles and hysteresis plots for each frequency were produced after each trial, as seen in Figure 9.

Just as with the monotonic loading, the worst-case compressive normal loads were $250 \mathrm{lb}_{\mathrm{f}}$ and $1500 \mathrm{lb}_{\mathrm{f}}$ for the passive and active rollers. The equivalent viscous damping coefficients, $C_{e q}$, were determined by equating the energy dissipated in a single test cycle to the energy that would be dissipated by a purely viscous damper undergoing the same test cycle, such that

$$
C_{e q}=W_{d} / \pi \omega X^{2}
$$




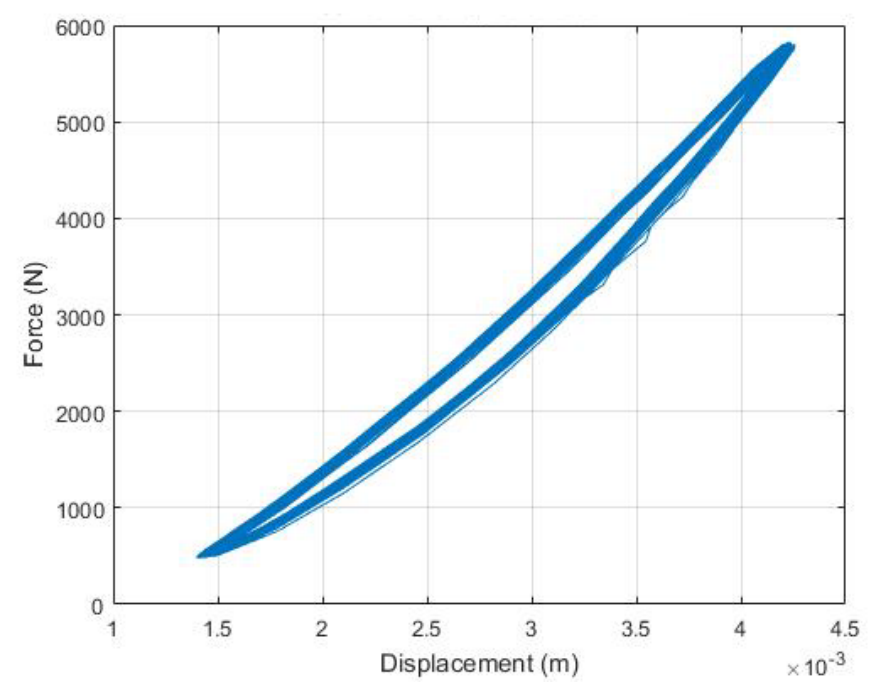

Fig. 9: Active roller hysteresis at $1 \mathrm{~Hz}$.

where $W_{d}$ is the energy dissipated through damping per cycle, $\omega$ is the excitation frequency, and $X$ is the displacement amplitude.

The damping work, $W_{d}$, was found by integrating the area within the hysteresis curve for every cycle at each frequency. Since the MTS was set to force control, the displacement amplitude, $X$, was taken from the recorded data. The peaks from the sinusoidal plot of displacement with respect to time were averaged and then the damping coefficient was calculated for every cycle. The mean of the coefficients was taken to be the damping coefficient at the given frequency. By acquiring the damping constants at several loading frequencies, the initial roller damping curves were obtained as seen in Figure 10.
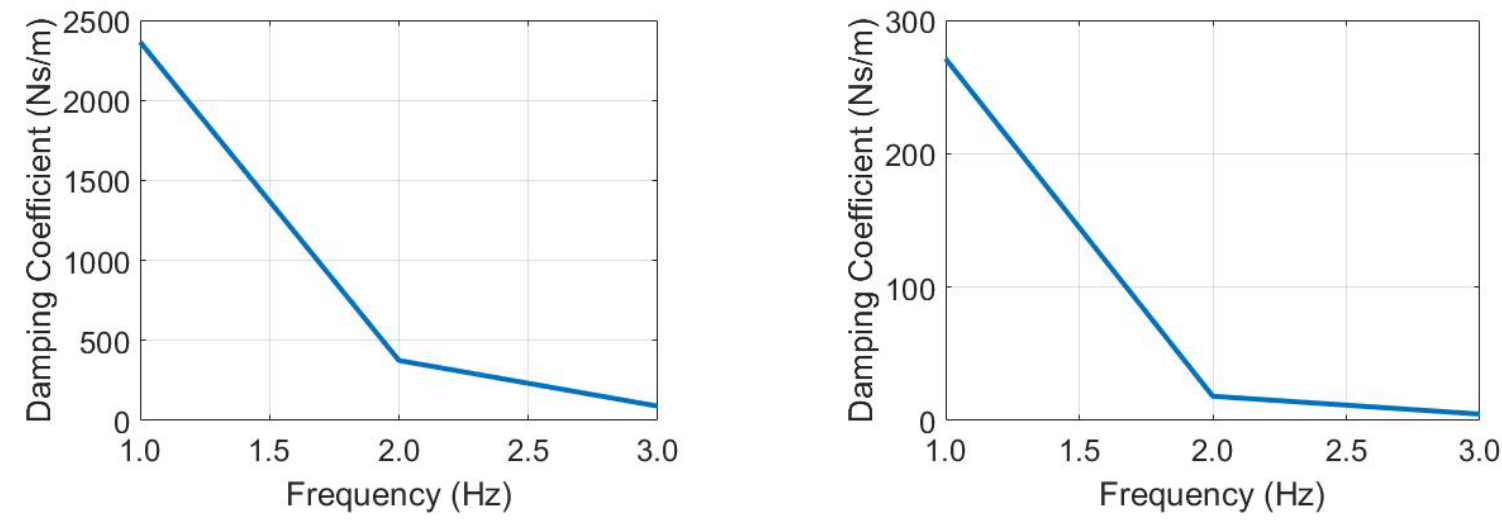

Fig. 10: Active roller damping curve (left) and passive roller damping curve (right).

\section{Results}

Using the parameters determined using the methods described in the previous sections, the equivalent spring constant was calculated for the lower passive wheels and also for the halo passive wheels; and the results are summarized in Tables 1 and 2 , for the lower and halo wheels, respectively.

\section{Conclusions and Future Work}

Structural and elastic parameters that govern the function of the Atlas system were determined by treating connecting components as springs. Through the use of FEA, spring constants for the structural components were determined. Various experiments were also conducted in order to characterize the properties of the mecanum wheel rollers. An MTS and a hydraulic press were used to subject individual rollers to monotonic and cyclic loading conditions such that their properties could be determined. 
Table 1: Lower passive wheel spring constants.

\begin{tabular}{|c|c|}
\hline Component & Spring Constant [N/m] \\
\hline \hline Roller $(2$ in parallel) & $8.66 \times 10^{5}$ \\
\hline Roller support & $6.68 \times 10^{7}$ \\
\hline Aluminum base & $1.17 \times 10^{7}$ \\
\hline Equivalent & $7.97 \times 10^{5}$ \\
\hline
\end{tabular}

Table 2: Halo passive wheel spring constants.

\begin{tabular}{|c|c|}
\hline Component & Spring Constant $[\mathrm{N} / \mathrm{m}]$ \\
\hline \hline Roller & $4.33 \times 10^{5}$ \\
\hline Aluminum structure & $2.40 \times 10^{8}$ \\
\hline Equivalent & $4.32 \times 10^{5}$ \\
\hline
\end{tabular}

These parameters were then available for use with a nine-degree-of-freedom model representing the translational dynamics of the Atlas simulator system.

The structural stiffnesses and material parameters that were determined offer an excellent set of initial values. Work will continue, by using the model to determine the sensitivity of the system behaviour to the stiffness and damping properties. Based on the results, it may be determined to validate the the structural stiffness values experimentally and/or to refine the measured material property values by performing additional tests - particularly for the damping values since they were found to be highly excitation frequency dependent. As the full-scale system becomes operational over the next several months, and given that it is quite extensively instrumented, additional opportunities will exist for validating the dynamic model and its constituent parameter values.

The next significant step for the dynamic model will be to include the rotational dynamics of all three modelled components: the sphere, the halo, and the interface platform; as well as to begin collecting model validation data using the full-scale Atlas prototype simulator.

\section{Acknowledgements}

The authors are grateful for financial support for the project provided by the Carleton University Department of Mechanical and Aerospace Engineering, the Carleton University Engineering Student Equipment Fund (CUESEF), and the Carleton Student Engineering Society (CSES). The authors acknowledge the technical support of Laboratory Technologist Steve Truttmann and members of the Machining Laboratory in supporting the experimental portion of this work. The authors also appreciate the helping hands and supportive environment provided by the 2016-17 CUSP team.

\section{References}

[1] Z. Copeland, B. Jung, M.J.D. Hayes, R.G. Langlois. "Full-scale Atlas Motion Platform: Structure, Actuation, and Control", International Journal of Mechanisms and Robotic Systems, vol. 3, n. 2/3, pp. 94-112, 2016.

[2] J.J. Plumpton, M.J.D. Hayes, R.G. Langlois, B.V. Burlton. "Atlas Motion Platform Mecanum Wheel Jacobian in the Velocity and Static Force Domains", Transactions of the Canadian Society for Mechanical Engineering, Vol. 38, No. 2, pp.251-261, 2014.

[3] K. Leigh, Interface Platform Stress Analysis. CUSP Technical Report No. DR-ACT-KL.17.02, Carleton Univ., 2017.

[4] J. Thevenot MRC and Dynamic Loading Model. CUSP Technical Report No. DR-ACT-JT.17.02, Carleton Univ., April 2017.

[5] Abaqus 6.14 Documentation. Dassault Systemes, 2014. (http://50.16.225.63/v6.14/books/key/default.htm?startat=ch11 .html)

[6] Creo 2.0 Quick Reference Guide. TriStar Inc., 2012. (http://www.tristar.com/product/creo-2-0-qrg/)

[7] J. van Waarden, Passive Wheel Mounting. CUSP Technical Report No. DR-STR-JvW.14.03, Carleton Univ., April 2014.

[8] M. Rappoport, Investigating Castor Roller Properties. CUSP Technical Report No. DR-ACT-MRA.17.02, Carleton Univ., 2017. 